\title{
HYPERTROPIA CORRECTED BY CHANGES OF THE LATERAL RECTUS INSERTION*
}

\author{
BY \\ JOHN FOSTER and E. C. PEMBERTON
}

EIGHT years ago we published 26 cases of concomitant convergent squint (Foster and Pemberton, 1946), which had been treated conventionally by advancement of the lateral rectus and recession of the medial rectus, plus an elevation or depression of either the medial or lateral rectus insertion, to correct a small vertical error. Our results showed that:

(1) elevation of a lateral rectus insertion raised the eye.

(2) depression of a lateral rectus insertion lowered the eye.

(3) cyclophoria occurred in two cases only and was slight.

We concluded that the operation had some justification when a vertical error was constant and less than 11 prism dioptres at all horizontal angles on the synoptophore.

Alvaro (1950) had extended the procedure by raising the insertion of both medial and lateral recti simultaneously in the same eye.

As we still encounter colleagues who not only consider that raising a lateral rectus lowers the eye and are prepared to prove it geometrically, we feel that our first series of cases failed to produce complete conviction and that the publication of a further series, 29 in number, might be timely. The cases recorded in the Table overleaf include all those we have treated in this way since 1948, the relatively small number being due to an increased readiness to operate on the obliques with an improvement in the technique of such operations. It will be observed that:

(1) the new results are "on all fours" with the first series-raising of the external rectus raises the eye;

(2) Alvaro found that raising or lowering of both lateral recti simultaneously, alters the position of the eye in a way consistent with our findings;

(3) cyclophoria is absent in this series.

It is interesting to note that the raising of both lateral recti produces less effect than we had expected, in view of the effect gained from the operation on one muscle.

Although the surgery of the obliques has advanced considerably in Great Britain, we still feel that there is a place for this operation, and our views as to its effect on the position of the eye remain unchanged.

\section{REFERENCES}

Foster, J., and Pemberton, E. C. (1946). British Journal of Ophthalmology, 30, 88. Alvaro, M. E. (1950). Ophthalmologica (Basel), 120, 191. 


\begin{tabular}{|c|c|c|c|c|}
\hline $\begin{array}{l}\text { Serial } \\
\text { Case } \\
\text { No. }\end{array}$ & Operation & $\begin{array}{c}\text { Pre-operative } \\
\text { Measurement } \\
\text { (with correction) }\end{array}$ & $\begin{array}{c}\text { Post-operative } \\
\text { Measurement } \\
\text { (with correction) }\end{array}$ & $\begin{array}{c}\text { Subsequent } \\
\text { Measurement } \\
\text { (with correction } \\
1-3 \text { mths later) }\end{array}$ \\
\hline 1 & 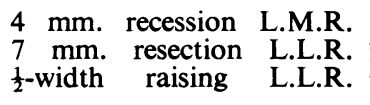 & $+15^{\circ} \mathrm{R} / \mathrm{L} 6^{\Delta}$ & $\begin{array}{l}\text { FRE }-10^{\circ} \mathrm{L} / \mathrm{R} 2^{\Delta} \\
\text { FLE }-14^{\circ} \mathrm{L} / \mathrm{R} 2 \Delta\end{array}$ & $0^{\circ} \mathrm{L} / \mathbf{R}$ \\
\hline 2 & $\begin{array}{lccc}4 & \text { mm. } & \text { recession } & \text { R.M.R. } \\
5 & \text { mm. } & \text { resection } & \text { R.L.R. } \\
\frac{1}{2} \text {-width } & \text { raising } & \text { R.L.R. }\end{array}$ & $\begin{array}{l}\text { FRE }+15^{\circ} \mathrm{L} / \mathrm{R} 8^{\triangle} \\
\text { FLE }+18^{\circ} \mathrm{L} / \mathrm{R} 8^{\Delta}\end{array}$ & FEE $-6^{\circ} \mathrm{L} / \mathrm{R} \quad 7^{\Delta}$ & $+3^{\circ} \theta$ \\
\hline 3 & $\begin{array}{l}5 \mathrm{~mm} \text {. recession L.M.R. } \\
7 \mathrm{~mm} \text {. resection and } \\
4 \mathrm{~mm} . \text { advancement L.L.R. } \\
\frac{1}{2} \text {-width lowering L.L.R. }\end{array}$ & $+31^{\circ} \mathbf{L} / \mathbf{R} 7 \Delta$ & $-3^{\circ} \mathrm{R} / \mathrm{L} 3^{\Delta}$ & \\
\hline 4 & $\begin{array}{lll}4 \mathrm{~mm} . & \text { recession } & \text { R.M.R. } \\
\frac{1}{2} \text {-width } & \text { lowering } & \text { R.M.R. } \\
6 \mathrm{~mm} . & \text { resection } & \text { R.L.R. } \\
\frac{1}{2} \text {-width } & \text { lowering } & \text { R.L.R. }\end{array}$ & $+17^{\circ} \mathrm{R} / \mathrm{L} 8^{\Delta}$ & $+6^{\circ} \theta$ & $+3^{\circ} \mathrm{L} / \mathbf{R} 2$ \\
\hline 5 & $\begin{array}{lcc}4 \mathrm{~mm} . & \text { recession } & \text { L.M.R. } \\
\frac{1}{2} \text {-width } & \text { raising } & \text { L.M.R. } \\
4 \quad \text { mm. } & \text { resection } & \text { L.L.R. } \\
\frac{1}{2} \text {-width } & \text { raising } & \text { L.L.R. }\end{array}$ & $+14^{\circ} \mathrm{R} / \mathrm{L} 8^{\Delta}$ & $+12^{\circ} \mathrm{L} / \mathrm{R} 4^{\Delta}$ & $\begin{array}{l}\text { Without glasses } \\
-3^{\circ} \mathrm{L} / \mathrm{R} 4\end{array}$ \\
\hline 6 & $\begin{array}{lcc}4 \text { mm. } & \text { recession } & \text { R.M.R. } \\
\frac{1}{2} \text {-width } & \text { raising } & \text { R.M.R. } \\
8 \text { mm. } & \text { resection } & \text { R.L.R. } \\
\frac{1}{2} \text {-width } & \text { raising } & \text { R.L.R. }\end{array}$ & $\begin{array}{l}\text { No glasses worn } \\
\text { FRE }+28^{\circ} \mathrm{L} / \mathrm{R} 15^{\Delta} \\
\text { FLE }+25^{\circ} \mathrm{L} / \mathrm{R} 13 \Delta\end{array}$ & $\begin{array}{l}\text { No glasses } \\
\text { FRE }+7^{\circ} \text { worn } \\
\text { FLE } 15 \Delta \\
\text { FL } 2^{\circ} \text { L/R } 13 \Delta\end{array}$ & $\begin{array}{l}\text { No glassess wor } \\
\text { FEE }+3^{\circ} \mathrm{L} / \mathrm{R} 8^{\circ}\end{array}$ \\
\hline 7 & 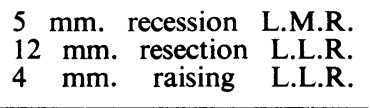 & $+44^{\circ} \mathrm{R} / \mathrm{L} \quad 7^{\Delta}$ & $+10^{\circ} \mathrm{L} / \mathrm{R} 5^{\Delta}$ & $+4^{\circ} \mathrm{L} / \mathrm{R} 5$ \\
\hline 8 & 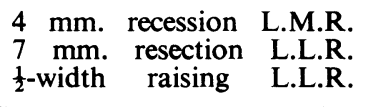 & $+19^{\circ} \mathbf{R} / \mathbf{L} 6^{\Delta}$ & $+6^{\circ} \mathrm{L} / \mathrm{R} 6^{\Delta}$ & \\
\hline 9 & $\begin{array}{cccc}5 & \text { mm. } & \text { resection } & \text { R.M.R. } \\
2 & \text { mm. } & \text { raising } & \text { R.M.R. }\end{array}$ & FRE $-13^{\circ} \mathrm{L} / \mathrm{R} 11^{\triangle}$ & $\mathrm{FEE}-2^{\circ} \mathrm{L} / \mathrm{R} 6^{\circ}$ & FEE $-5^{\circ} \mathrm{L} / \mathrm{R} 3$ \\
\hline 10 & 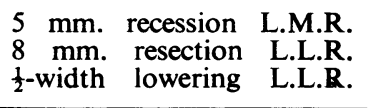 & $+27^{\circ} \mathrm{L} / \mathrm{R} 6^{\Delta}$ & $+20^{\circ} \mathbf{L} / \mathbf{R} 6^{\Delta}$ & $+\mathbf{5}^{\circ} \mathbf{L} / \mathbf{R}$ \\
\hline 11 & $\begin{array}{ccc}3 \mathrm{~mm} . & \text { recession } & \text { L.M.R. } \\
\frac{1}{2} \text {-width } & \text { lowering } & \text { L.M.R. } \\
4 \mathrm{~mm} . & \text { resection } & \text { L.L.R. } \\
\frac{1}{2} \text {-width } & \text { lowering } & \text { L.L.R. }\end{array}$ & $\begin{array}{ll}\text { No } & \text { glasses worn } \\
& +18^{\circ} \mathrm{L} / \mathrm{R} 9^{\Delta}\end{array}$ & $\begin{array}{ll}\text { No } & \text { glasses } \\
& -3^{\circ} \mathbf{L} / \mathbf{R} 2^{\Delta} \Delta\end{array}$ & $\begin{array}{l}\text { No glasses worn } \\
-2^{\circ} \mathrm{R} / \mathrm{L} 2 \Delta\end{array}$ \\
\hline 12 & 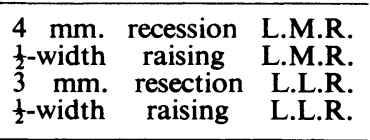 & $+16^{\circ} \mathrm{R} / \mathrm{L} 6^{\Delta}$ & $+9^{\circ} \mathrm{R} / \mathrm{L} \quad 2^{\Delta}$ & $+9^{\circ} \mathbf{R} / \mathbf{L}$ \\
\hline 13 & $\begin{array}{lcc}5 \text { mm. } & \text { recession } & \text { L.M.R. } \\
\frac{1}{4} \text {-width } & \text { raising } & \text { L.M.R. }\end{array}$ & $+12^{\circ} \mathrm{R} / \mathrm{L} 4^{\Delta}$ & $+5^{\circ} \mathrm{R} / \mathrm{L} 1 \Delta$ & $+4^{\circ} \theta$ \\
\hline 14 & $\begin{array}{lccc}5 & \text { mm. } & \text { recession } & \text { L.M.R. } \\
4 & \text { mm. } & \text { resection } & \text { L.L.R. } \\
\frac{1}{2} \text {-width } & \text { raising } & \text { L.L.R. }\end{array}$ & 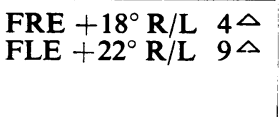 & $+8^{\circ} \mathrm{L} / \mathrm{R} 17^{\Delta}$ & $\begin{array}{l}\text { FRE }+9^{\circ} \text { L/R } 13 \Delta \\
\text { FLE }+13^{\circ} \mathbf{L} / \mathbf{R} 9 \Delta\end{array}$ \\
\hline
\end{tabular}




\begin{tabular}{|c|c|c|c|c|}
\hline $\begin{array}{l}\text { Serial } \\
\text { Case } \\
\text { No. }\end{array}$ & Operation & $\begin{array}{c}\text { Pre-operative } \\
\text { Measurement } \\
\text { (with correction) }\end{array}$ & $\begin{array}{c}\text { Post-operative } \\
\text { Measurement } \\
\text { (with correction) }\end{array}$ & $\begin{array}{c}\text { Subsequent } \\
\text { Measurement } \\
\text { (with correction } \\
1-3 \text { mths later) }\end{array}$ \\
\hline 15 & 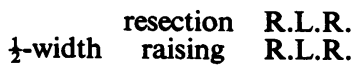 & $+15^{\circ} \mathrm{L} / \mathrm{R} 8^{\Delta}$ & $+15^{\circ} \mathrm{L} / \mathrm{R} 8^{\Delta}$ & $+7^{\circ} \mathbf{L} / \mathbf{R} 4^{\Delta}$ \\
\hline 16 & 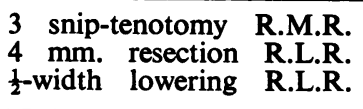 & $\begin{array}{l}\text { FRE }+5^{\circ} \text { R/L } 8^{\Delta} \\
\text { FLE }+5^{\circ} \text { R/L } 10^{\Delta}\end{array}$ & $\begin{array}{l}\text { FRE }-8^{\circ} \mathrm{R} / \mathrm{L} 3^{\Delta} \\
\text { FLE }-8^{\circ} \mathrm{R} / \mathrm{L} 6^{\Delta}\end{array}$ & $0^{\circ} \theta$ \\
\hline 17 & $\begin{array}{lcrr}4 & \mathrm{~mm} . & \text { recession } & \text { R.M.R. } \\
8 & \mathrm{~mm} . & \text { resection } & \text { R.L.R. } \\
3 & \mathrm{~mm} . & \text { lowering } & \text { R.L.R. }\end{array}$ & $\begin{array}{l}\text { FRE }+17^{\circ} \text { R/L } 5 \Delta \\
\text { FLE }+28^{\circ} \text { R/L } 9 \Delta\end{array}$ & $\mathrm{FEE}+8^{\circ} \mathrm{L} / \mathrm{R} 5^{\Delta}$ & $\begin{array}{l}\text { Without glasses } \\
\text { FRE }-4^{\circ} L / R \quad 7 \Delta \\
\text { FLE }+5^{\circ} \text { L/R } 7 \Delta\end{array}$ \\
\hline 18 & $\begin{array}{lccc}4 & \text { mm. } & \text { recession } & \text { R.M.R. } \\
4 & \text { mm. } & \text { resection } & \text { R.L.R. } \\
\frac{1}{2} \text {-width } & \text { raising } & \text { R.L.R. }\end{array}$ & $+9^{\circ} \mathrm{L} / \mathrm{R} 6^{\Delta}$ & $+5^{\circ} \theta$ & $-6^{\circ} \theta$ \\
\hline 19 & 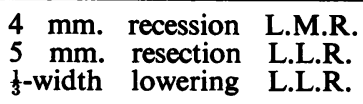 & $\begin{array}{ll}\text { No } & \text { glasses worn } \\
& +16^{\circ} \mathrm{L} / \mathrm{R} \quad 3 \Delta\end{array}$ & No $\begin{array}{c}\text { glasses } \\
+8^{\circ}\end{array}$ & No $\underset{+5^{\circ} \theta}{\text { glasses worn }}$ \\
\hline 20 & 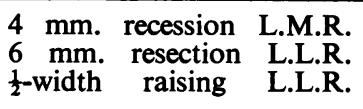 & $+18^{\circ} \mathrm{R} / \mathrm{L} \quad 9^{\triangle}$ & $-2^{\circ} \mathrm{R} / \mathrm{L} 2^{\Delta}$ & $+4^{\circ} \mathbf{L} / \mathbf{R} \cdot \mathbf{\Delta}$ \\
\hline 21 & $\begin{array}{cccc}5 & \mathrm{~mm} . & \text { recession } & \text { R.M.R. } \\
8 & \mathrm{~mm} . & \text { resection } & \text { R.L.R. } \\
\frac{1}{2} \text {-width } & \text { lowering } & \text { R.L.R. }\end{array}$ & $\begin{array}{l}\text { FRE }+25^{\circ} \text { R/L } 6 \Delta \\
\text { FLE }+28^{\circ} \text { R/L } 13^{\Delta}\end{array}$ & $+15^{\circ} \mathrm{R} / \mathrm{L} 10^{\Delta}$ & $+11^{\circ} \theta$ \\
\hline 22 & $\begin{array}{lcc}4 \mathrm{~mm} . & \text { recession } & \text { L.M.R. } \\
\frac{1}{2} \text {-width } & \text { lowering } & \text { L.M.R. } \\
5 \mathrm{~mm} . & \text { resection } & \text { L.L.R. } \\
\frac{1}{2} \text {-width } & \text { lowering } & \text { L.L.R. }\end{array}$ & $\begin{array}{l}\text { FRE }+21^{\circ} \mathrm{L} / \mathrm{R} 7^{\Delta} \\
\mathrm{FLE}+22^{\circ} \mathrm{R} / \mathrm{L} 5^{\Delta}\end{array}$ & $+15^{\circ} \mathrm{L} / \mathrm{R} 5^{\Delta}$ & $\begin{array}{l}\text { FRE }+17^{\circ} \mathbf{L} / \mathbf{R}^{\circ \Delta} \\
\text { FLE }+15^{\circ} \diamond\end{array}$ \\
\hline 23 & 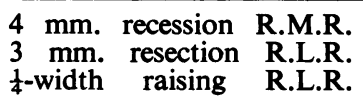 & $+8^{\circ} \mathbf{L} / \mathbf{R} 3^{\Delta}$ & $+8^{\circ} \theta$ & $+8^{\circ} \theta$ \\
\hline 24 & 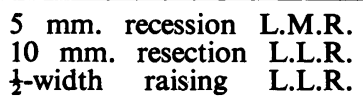 & $+30^{\circ} \mathrm{R} / \mathrm{L} 8^{\Delta}$ & $+8^{\circ} \mathrm{R} / \mathrm{L} 2^{\Delta}$ & $\begin{array}{l}\text { After further recession } \\
\text { R.M.R. Own glasses } \\
=0^{\circ} \text { L/R } 3 \Delta\end{array}$ \\
\hline 25 & $\begin{array}{l}5 \mathrm{~mm} \text {. recession R.M.R. } \\
5 \mathrm{~mm} \text {. resection R.L.R. } \\
6.5 \mathrm{~mm} \text {. lowering } \\
\text { R.L.R. }\end{array}$ & $\begin{array}{l}\text { No glasses worn } \\
\text { FRE }+30^{\circ} \mathrm{R} / \mathrm{L} 13 \Delta \\
\text { FLE }+26^{\circ} \mathrm{R} / \mathrm{L} 13 \Delta\end{array}$ & $\begin{array}{l}\text { No glasses worn } \\
\text { FRE }+14^{\circ} \mathrm{L} / \mathrm{R}^{2} 2^{\Delta} \\
\text { FLE }+14^{\circ} \bullet\end{array}$ & $\begin{array}{l}\text { No glasses worn } \\
\text { FEE }+2^{\circ} \mathrm{L} / \mathrm{R} \quad 1 \Delta\end{array}$ \\
\hline 26 & $\begin{array}{l}\text { Recession and lowering } \\
\text { L.L.R. }\end{array}$ & $\begin{array}{l}\text { No glasses worn } \\
\text { FRE }-8^{\circ} \mathrm{L} / \mathbf{R} \quad 6 \Delta \\
\text { FLE }-4^{\circ} \mathrm{L} / \mathbf{R} 2^{\Delta}\end{array}$ & $\begin{array}{l}\text { No glasses } \\
\text { FEE }+1-13^{\circ} \\
\end{array}$ & $\begin{array}{l}\text { No glasses worn } \\
+1^{\circ}-1^{\circ} \stackrel{ }{\circ}\end{array}$ \\
\hline 27 & 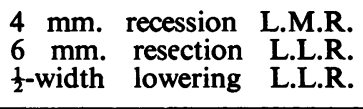 & $+17^{\circ} \mathrm{L} / \mathrm{R} 3^{\Delta}$ & $+2^{\circ} \theta$ & $-4^{\circ} \theta$ \\
\hline 28 & $\begin{array}{l}4 \mathrm{~mm} \text {. recession R.M.R. } \\
\text { whole width raising R.M.R. } \\
8 \mathrm{~mm} \text {. resection R.L.R. } \\
\text { whole width raising R.L.R. }\end{array}$ & $\begin{array}{l}\text { FRE }+22^{\circ} \text { L/R } 16^{\Delta} \\
\text { FLE }+22^{\circ} \text { L/R } 12^{\Delta}\end{array}$ & FEE $0^{\circ} \mathrm{R} / \mathrm{L} \quad 1-2 \Delta$ & \\
\hline 29 & $\begin{array}{ccc}5 \mathrm{~mm} . & \text { recession } & \text { R.M.R. } \\
\frac{1}{2} \text {-width } & \text { raising } & \text { R.M.R. } \\
6 \text { mm. } & \text { resection } & \text { R.L.R. } \\
\frac{1}{2} \text {-width } & \text { raising } & \text { R.L.R. }\end{array}$ & $\begin{array}{l}\text { FRE }+23^{\circ} \mathrm{L} / \mathrm{R} 7^{\Delta} \\
\text { FLE }+23^{\circ} \mathrm{L} / \mathrm{R} 4^{\Delta}\end{array}$ & $\mathrm{FEE}+10^{\circ} \bullet$ & \\
\hline
\end{tabular}

\section{Key to TABle}

$\begin{array}{llll}\text { L.M.R. } & \text { Left medial rectus } & \text { F.R.E. } & \text { Fixing right eye } \\ \text { L.L.R. } & \text { Left lateral rectus } & \text { F.L.E. } & \text { Fixing left eye } \\ \text { R.M.R. } & \text { Right medial rectus } & \text { F.E.E. } & \text { Fixing each eye } \\ \text { R.L.R. } & \text { Right lateral rectus } & - & \text { No vertical error }\end{array}$

